\section{Standardized Uniqueness: Oxymoron or Vision
of the Future?}

Erik Duval

Katholieke Universiteit Leuven

\section{Wayne Hodgins}

Autodesk Inc.

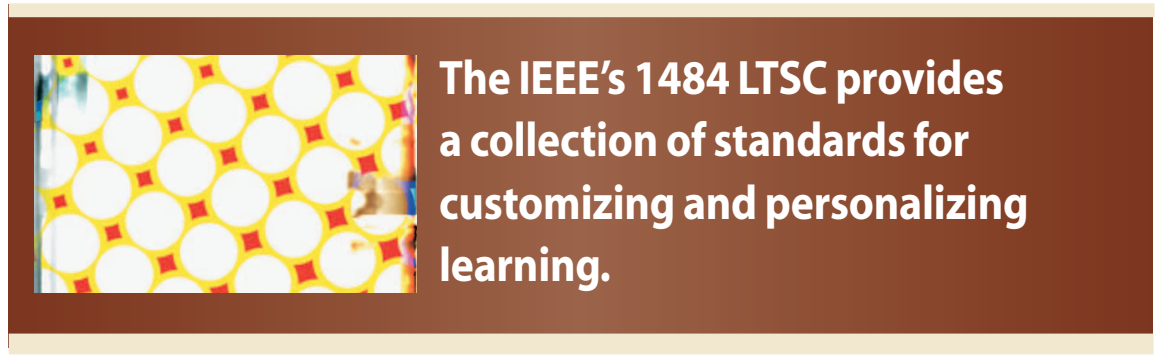

I magine a world where everyone experiences "just right" learning: just the right content, at just the right time, in just the right context, in just the right medium. Imagine this massively customized and personalized "me-learning" for each of the 6.4 billion unique individuals in the world, 24/7.

Our vision for the future of learning is one of mass customization and, ultimately, personalization. While it might be difficult to imagine such a world, it is perhaps even more difficult to realize that a fundamental enabler of such uniqueness and personalization is standards.

"Surely, you must be joking" is an expected response, but this vision is a fundamental driver for a collection of standards within the IEEE 1484 Learning Technology Standards Committee.

The LTSC's scope is to develop standards for learning technology-not for learning itself. The first LTSC standard, Learning Object Metadata, was completed in 2002 and several more standards have followed since then.

\section{THE LOM STANDARD}

In the LOM context, learning objects are small, reusable content units relevant for learning. Metadata describes the learning objects' attributes, making it easier to find, evaluate, acquire, assemble, and deploy relevant content (E. Duval et al., "Metadata Principles and Practicalities," D-lib Magazine, Apr. 2002, pp. 1-16).

As Figure 1 shows, the core of the multipart LOM 1484.12 standard is a conceptual structure for a metadata instance based on a hierarchy of nine categories of metadata elements.

For every metadata element, the 1484.12.1 LOM data model defines the number of values allowed, indicates whether the order of values is significant, and identifies the set of allowed values and the data type. In this context, data types are structures based on other standards such as ISO 8601 or ISO/IEC 10646 or specifications such as vCard.

LOM instances can include extensions to the base schema. The explicit goal is to evolve the standard when experience in practice demonstrates a widespread adoption of such extensions.

In practice, communities such as the US Advanced Distributed Learning initiative or the European ARIADNE Foundation translate their needs and requirements into application profiles that make metadata elements mandatory, add extensions, or restrict value spaces.

By adopting LOM, these communities can exchange metadata at a basic level of interoperability. Through the application profile, they can meet their internal requirements at a higher level, for example, by requiring that an object has a title in all the official European languages.

LOM accommodates multilinguality for both the learning object content and the metadata. For example, every string value can be repeated for multilanguage metadata instances.

In 2005, IEEE 1484.12.3, the standard for the XML binding of LOM, was finalized as a set of XML schemas that a particular community can configure to satisfy its needs.

\section{THE KEY TO SUCCESS}

Accredited standards serve a purpose and make a profound difference. History has shown that the takeoff point for many innovations includes the adoption of common standards. Examples include railway track gauge, telephone dial tones, videotape formats, e-mail protocols, the Internet, and the World Wide Web. Without the adoption of common standards, the market stalls. Consider the historic battle between VHS and Beta that hindered the eventual explosion of the video industry, or more current examples such as the lack of common stan- 
dards for DVDs (Blue-Ray versus HD DVD) and instant messaging.

Today more than ever, to be true enablers, standards must be flexible and adaptive so that they can be increasingly useful as circumstances inevitably change-often unexpectedly so.

In the work on LOM and other LTSC standards, a key attribute to success has emerged: flexibility-keep the standards small and modular.

LOM provides an example of such flexibility, offering modularity in two significant ways. Every LOM metadata element is optional. Reaching agreement on this purposeful decision took several years. LOM effectively standardizes how to structure metadata about learning objects, not which metadata elements to include. Many successful standards incorporate this same flexibility attribute, including HTML, where most tags are also optional. Yet these are true standards in that once the authors make a decision about what to express, the standard provides a clear, consistent, and unambiguous way of doing so.

LOM ensures maximum flexibility and longevity because it is a multipart standard. To ensure maximum modularity, interoperability, choice, and adaptability, we separated the standard for the individual metadata elementsLOM's data model (1484.12.1) -from the technical binding or expression of these metadata elements with XML (1484.12.3). We believe this modularity also accounts for why LOM has thus far exceeded all expectations of success as it has been globally and universally adopted, adapted, and implemented in many unexpected ways and applications.

\section{WHERE TO FROM HERE?}

Achieving LOM's full potential requires meeting the challenge of developing and promoting the adoption of tools that make the metadata completely transparent to end users ("Making Metadata Go AwayHiding Everything But the Benefits," Proc. Int'l Conf. Dublin Core and Metadata Applications, 2004, pp. 29-

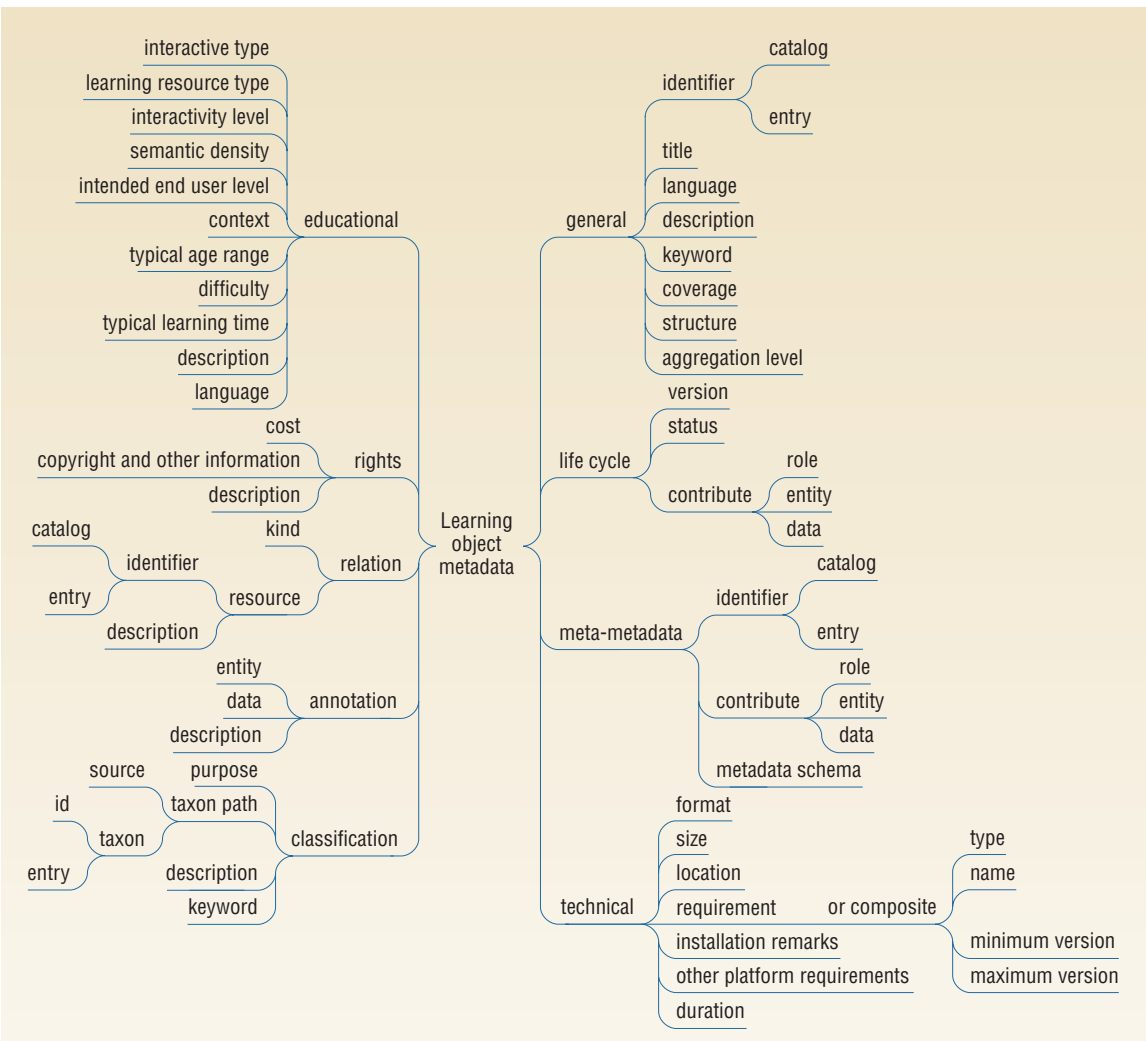

Figure 1. Learning object metadata structure. This structure is based on a hierarchy of nine categories of metadata elements.

35, www.slais.ubc.ca/PEOPLE/faculty/ tennis-p/dcpapers2004/Paper_15.pdf). Similar to early Web authoring tools that showed the details of HTML tags, many early LOM tools were basically electronic forms that closely followed the LOM standard. Only recently have tools become available that automate the metadata authoring process, using personal profiles of metadata and contextual information, as well as mining the learning object for metadata.

Similarly, search applications increasingly rely on a simple Google-like interface to process queries over a federated worldwide network of repositories. For end users, boundaries-whether physical, technical, or organizational-between the repositories are irrelevant, and they consider all of the material to be part of the global collection of learning content.

Taking this approach further, we can now evolve from searching to finding and presenting appropriate content when needed, without requiring an end user to know to request it. Context plays a paramount role here. For example, an RSS feed can include relevant content for a professor's academic course or personalized professional training can be part of an employee's desktop environment.

A similar evolution with related metadata formats like Dublin Core (from the more conventional library domain) or MPEG (from the audiovisual domain) mirrors the progress and deployment of metadata that the LOM standard supports. The evolution toward a Semantic Web offers tremendous potential for merging all metadata in a seamlessly integrated information environment. This is crucial for learning applications as material that was not originally purposely developed for learning can yield much relevant content.

s metadata becomes more pervasive, automatically generated, and transparently processed, we 
will find that, rather than identifying what, how, and who, searching will be more like the recent IEEE slogan: "Click less, find more." In the future, each situation's context will define the right content, and the information will increasingly find the user.

Although transforming this vision into reality requires substantial additional work, remarkable progress has been made during the past decade, including the wide-scale adoption of IEEE standards such as LOM.

As change tends to occur exponentially, it is possible to imagine a world where personalized "just right" learn- ing for all based on flexible metadata standards is the norm. Standardized uniqueness is clearly not an oxymoron but rather the secret to successfully realization of the vision of personalized learning for each unique individual, in unique situations, everyday, everywhere.

For those of us working on IEEE standards, ensuring that we are consistently improving our efforts becomes imperative as we strive to make this vision of learning a reality.

Erik Duval, professor at Katholieke Universiteit Leuven, is a technical editor of
IEEE LTSC 1484.12. Contact him at erik.duval@cs.kuleuven.ac.be.

Wayne Hodgins, director of worldwide learning strategies at Autodesk Inc., is chair of IEEE LTSC 1484.12 LOM. Contact him atwayne.hodgins@autodesk. com.

Editor: John Harauz, Jonic Systems Engineering, Inc., Willowdale, Ont., Canada; j.harauz@ieee.org

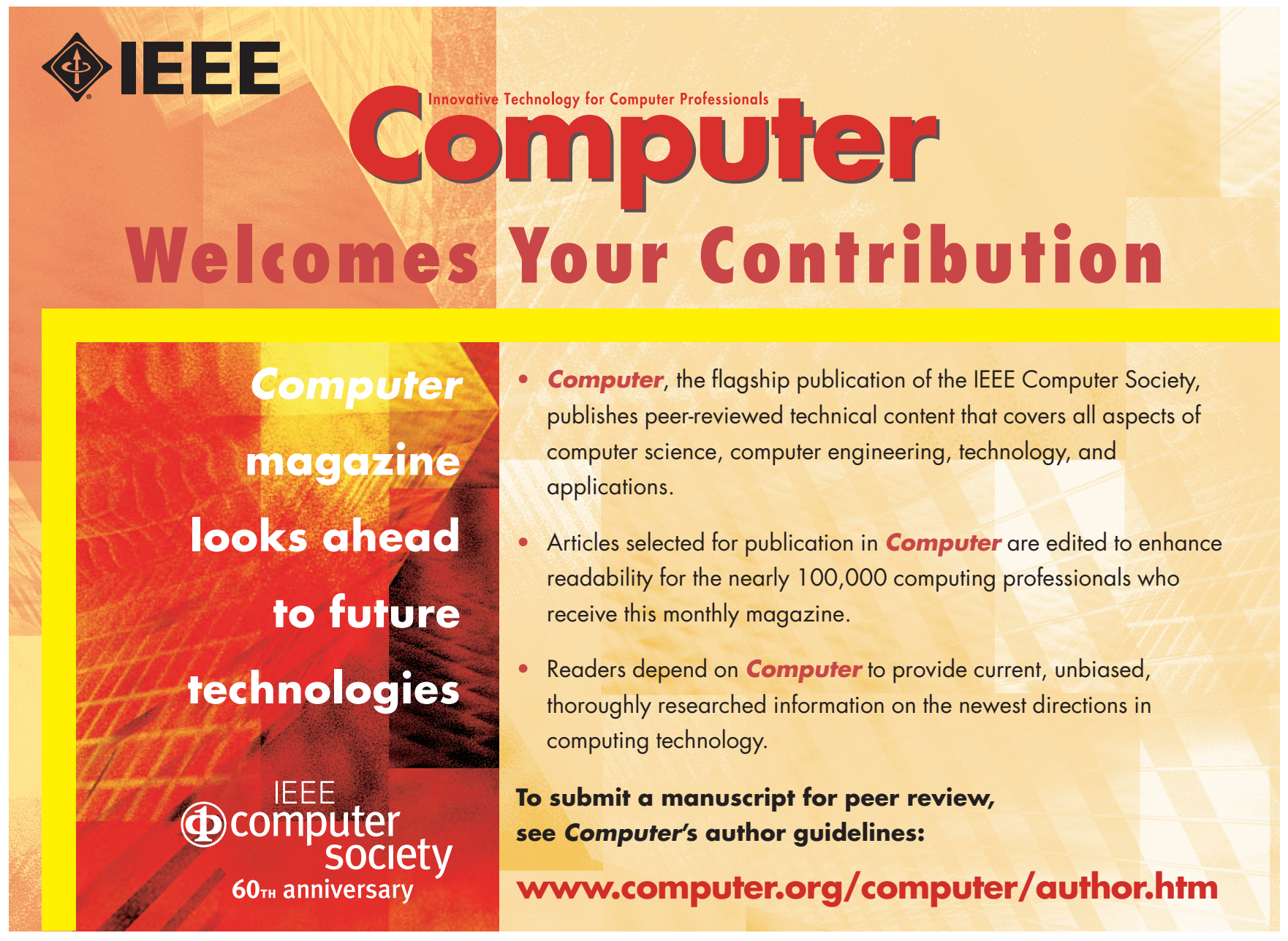

